\title{
SPIN EFFECTS IN LARGE RAPIDITY NEUTRAL PION PRODUCTION AT STAR
}

\author{
A. OGAWA FOR THE STAR COLLABORATION \\ Brookhaven National Laboratory, \\ Upton, NY 11973-5000, USA \\ E-mail: akio@bnl.gov
}

\begin{abstract}
First measurements of large Feynman $x\left(x_{F}\right)$ neutral pion production in polarized proton collisions at $\sqrt{s}=200 \mathrm{GeV}$ were reported previously. Cross section measurements at $\langle\eta\rangle=3.3$ and 3.8 and analyzing power $\left(A_{N}\right)$ measurements at $\langle\eta\rangle=3.8$ were completed for $0.3<x_{F}<0.6$. The cross section was found to be consistent with next-to-leading order perturbative QCD calculations. In that framework, the dominant subprocess is partonic collisions of valence quarks on low Bjorken- $x$ gluons. The $A_{N}$ was found to be large and positive and increasing with $x_{F}$, and consistent with phenomonological calculations based on the Collins effect, the Sivers effect and initial-state and final-state higher twist contributions. Subsequent data have been acquired with transverse polarized proton collisions at RHIC at $\sqrt{s}=200 \mathrm{GeV}$. The status of the analysis and preliminary results will be presented.
\end{abstract}

In the perturbative QCD picture, high $x_{F}$ hadron production in hadronhadron collisions probes asymmetric partonic collisions. It is dominated by the collisions of a large- $x$ quark on a low- $x$ gluon. Since the longitudinal polarization of large- $x$ quark is known to be large from polarized DIS experiments, this is an interesting place to study spin effects in the nucleon. Also it is an ideal probe for low- $x$ gluons because quarks directly couple with gluons.

An important question to address is whether fixed-order pQCD is appropriate to describe forward particle production in $\mathrm{p}+\mathrm{p}$ collisions at $\sqrt{s}=200$ $\mathrm{GeV}$. For $\sqrt{s} \leq 62 \mathrm{GeV}$, next-to-leading order pQCD severely underpredicts measured $\pi^{0}$ cross sections ${ }^{1}$. At $\sqrt{s}=200 \mathrm{GeV}$ and larger collision energies, there is quantitative agreement between NLO pQCD calculations and measured cross sections at mid-rapidity ${ }^{2}$. This agreement has been found to extend to $\pi^{0}$ production at $\langle\eta\rangle=3.8$ and $3.3^{3,4}$.

Further tests of the underlying dynamics responsible for forward particle production can be obtained from the study of particle correlations. In 
particular, strong azimuthal correlations of hadron pairs are expected when particle production arises from $2 \rightarrow 2$ parton scattering. The azimuthal correlations of hadron pairs separated by large $\Delta \eta$ were reported $^{5}$, and found to be consistent with PYTHIA predictions ${ }^{6}$.

In polarized proton-proton collisions, the transverse single spin asymmetry $\left(A_{N}\right)$ for the $p_{\uparrow}+p \rightarrow \pi^{0}+X$ reaction has been measured ${ }^{7,3}$ at $\sqrt{s}=20$ and $200 \mathrm{GeV}$ and found to be large. Semi-inclusive deep-inelastic lepton scattering experiments ${ }^{8,9}$ have reported measurements of transverse single-spin asymmetries which are significantly different from zero.

In perturbative QCD at leading twist with collinear factorization, $A_{N}$ has to be zero. If one takes a step beyond this simple scheme and allows partons to have transverse momentum $\left(k_{T}\right)$, there are 3 terms which can contribute to the asymmetry. The first is the Sivers effect ${ }^{10}$, which is an initial state correlation between parton $k_{T}$ and the transverse spin of the nucleon. The second is transverse polarization of the quark, or transversity, and the Collins effect ${ }^{11}$ in the fragmentation process. The third involves a correlation between $k_{T}$ and quark spin within the unpolarized nucleon, which is believed to be very small ${ }^{12}$. Calculations of twist-3 contributions ${ }^{13,14}$ also have 3 terms similar to the models with transverse momentum. While all these mechanisms can contribute at the same time, recent study ${ }^{15}$ shows that the Collins effect is suppressed due to cancellations in quantum phases.

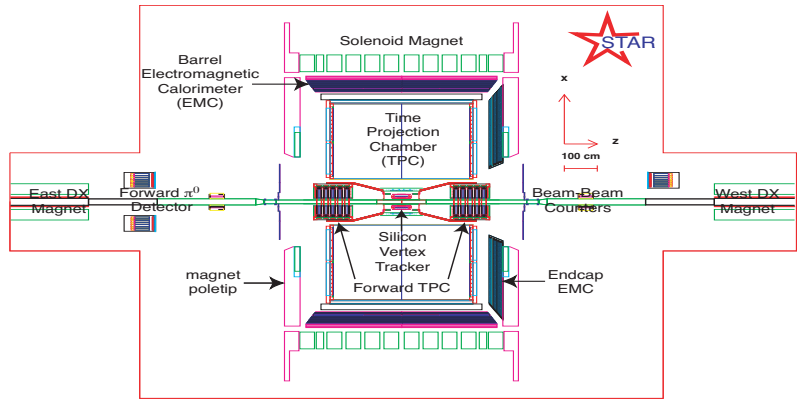

Figure 1. Top view of the STAR detector.

The Solenoid Tracker at RHIC (STAR) is a multipurpose detector at Brookhaven National Laboratory. One of its principle components is a time projection chamber (TPC and Forward-TPC) embedded in a $0.5 \mathrm{~T}$ solenoidal magnetic field used for tracking charged particles at $|\eta|<1.2$ and $2.8<|\eta|<3.8$. To extend the rapidity coverage, a forward $\pi^{0}$ detector 
(FPD) was installed at STAR, as shown in Fig.1. The FPD is a $7 \times 7$ array of $3.8 \mathrm{~cm} \times 3.8 \mathrm{~cm} \times 45 \mathrm{~cm}$ Pb-glass cells with individual photomultiplier tubes. For the 2003 RHIC run, both beam left and right detectors on the east side were installed, and a beam left detector was installed on the west side. The FPD provides triggering and reconstruction of neutral pions produced with $3.3<\eta<4.2$.

The energy calibration of the FPD is done using reconstructed $\pi^{0}$ mesons. The reconstruction method was extensively studied using GEANT Monte-Calro simulations ${ }^{16}$. The absolute energy scale is known to better than $2 \%$, and the reconstruction efficiency is found to be mostly determined by geometry. Photon conversion events primarily in the beam pipe producing hits in both the Forward-TPC and the FPD were used to determine the position of the FPD.
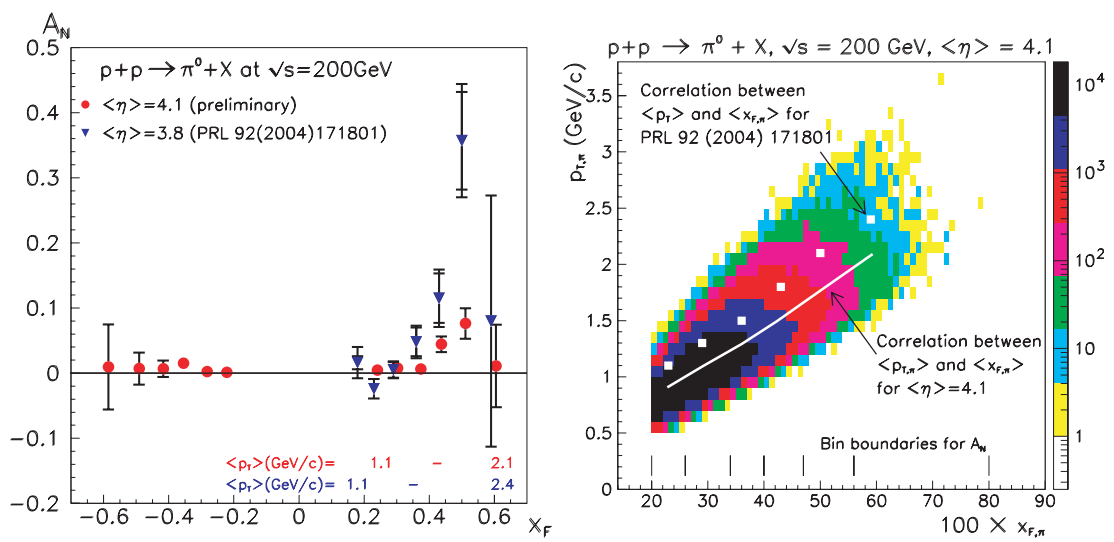

Figure 2. Left: Preliminary results on analyzing power of $p+p \rightarrow \pi^{0}+X$ as function of $x_{F}$ at $\langle\eta\rangle=4.1$ (circle), compared to published results at $\langle\eta\rangle=3.8$ (triangle). Right: $x_{F}$ and $p_{T}$ range of the data. The line shows the correlation between $\left\langle x_{F}\right\rangle$ and $\left\langle p_{T}\right\rangle$ for $\langle\eta\rangle=4$.1. The square dots shows the $\left\langle x_{F}\right\rangle$ and $\left\langle p_{T}\right\rangle$ for $<\eta>=3.8$.

In the 2003 RHIC run, $\mathrm{p}+\mathrm{p}$ collisions were studied at $\sqrt{s}=200 \mathrm{GeV}$ with average polarization $\sim 25 \%$ and integrated luminosity of $\sim 0.5 / p b$. The polarization was measured by the $\mathrm{pC}$ CNI polarimeter ${ }^{18}$. For the east side, the cross ratio method is used to obtain $A_{N}$. For the west side, the $A_{N}$ is obtained from spin dependent yields normalized by the BeamBeam Counter ${ }^{17}$. These 2 measurements are found to be consistent, and are combined. Positive (negative) $x_{F}$ is defined when the $\pi^{0}$ is observed 
with the same (opposite) longitudinal momentum as the polarized beam. Positive $A_{N}$ is defined as more $\pi^{0}$ going left of the upward polarized beam for both positive and negative $x_{F}$.

The preliminary results are shown in Fig.2. The $A_{N}$ for positive $x_{F}$ at $<\eta\rangle=4.1$ is found to be significantly non zero and positive, confirming earlier measurements. The first measurement of $A_{N}$ at negative $x_{F}$ has been done, and found to be consistent with zero. The negative $x_{F}$ results may give an upper limit on the gluon Sivers function ${ }^{15}$, which is currently unknown.

Future studies using the STAR FPD will include measurements of the $p_{T}$ dependence of $A_{N}$ at fixed $x_{F}$, di-hadron correlations to distinguish the Collins effect from the Sivers effect and $A_{L L}$ which is sensitive to gluon polarization at low- $x$.

\section{References}

1. C. Bourrely and J. Soffer, Eur. Phys. J. C36 371-374 (2004).

2. S. S. Adler et al., Phys. Rev. Lett. 91, 241803 (2003).

3. STAR Collaboration, Phys. Rev. Lett. 92 (2004) 171801.

4. L.C. Bland, hep-ex/0403012.

5. A. Ogawa, nucl-ex/0408004.

6. T. Sjöstrand, Comp. Phys. Commun. 82, 74 (1994).

7. A. Bravar et al., Phys. Rev. Lett. 772626 (1996) ; D. L. Adams et al., Phys. Rev. D53 4747 (1996)

8. A. Airapetian et al., Phys. Rev. Lett. 84, 4047 (2000); Phys. Lett. B 535, 85 (2002); 562, 182 (2003).

9. A. Bravar et al., Nucl. Phys. Proc. Suppl. 79, 520 (1999).

10. D. W. Sivers, Phys. Rev. D41 83 (1990)

11. J. C. Collins, S. F. Heppelmann, G. A. Ladinsky, Nucl. Phys. B396 161 (1993);

12. D. Boer, AIP Conf. Proc. 675 479-483 (2003).

13. J. Qiu and G. Sterman, Phys. Rev. D59, 014004 (1998).

14. Y. Koike, AIP Conf. Proc. 675, 449 (2003).

15. M. Anselmino et. al. hep-ph/0408356; U. D'Alesio, Proceedings of Spin2004

16. GEANT 3.21, CERN program library.

17. J. Kiryluk, AIP Conf. Proc. 675, 424 (2003).

18. O. Jinnouchi et al., AIP Conf. Proc. 675, 817 (2003). 